\title{
Hiperparatiroidismo primario: conceptos para el cirujano general
}

\author{
Primary hyperparathyroidism: concepts for the general surgeon \\ Gabriel Sánchez-De Guzmán $\mathbb{D}$, Aníbal Ariza² D
}

1 Médico, especialista en Cirugía general, subespecialista en Cirugía de Cabeza y cuello, Hospital Universitario San Ignacio, Bogotá, D.C., Colombia.

2 Médico, especialista en Cirugía general, Hospital Universitario San Ignacio; profesor instructor, Pontificia Universidad Javeriana, Bogotá, D.C., Colombia.

\section{Resumen}

Durante las últimas décadas, la incidencia del hiperparatiroidismo primario ha venido en aumento, muy probablemente relacionado con la mayor accesibilidad a los estudios diagnósticos; sin embargo, la forma más común de presentación clínica del hiperparatiroidismo primario es asintomática, en más del $80 \%$ de los pacientes. En la actualidad, es menos frecuente el diagnóstico por las complicaciones renales (urolitiasis) u óseas (osteítis fibrosa quística) asociadas.

Un tumor benigno de la glándula paratiroides (adenoma único), es la principal causa de esta enfermedad. Por tanto, su tratamiento usualmente es quirúrgico. A pesar de ello, no es frecuente el manejo de esta patología por el cirujano general.

En este artículo se revisan conceptos claves para el diagnóstico y manejo de esta enfermedad para el médico residente y especialista en Cirugía general.

Palabras clave: glándulas paratiroides; hiperparatiroidismo primario; hormona paratiroidea; neoplasias de las paratiroides; adenoma; hipercalcemia; paratiroidectomía.

\begin{abstract}
During the last decades, the incidence of primary hyperparathyroidism has been increasing, most probably related to the greater accessibility to diagnostic studies; however, the most common form of clinical presentation of primary hyperparathyroidism is asymptomatic in more than $80 \%$ of patients. Diagnosis is less frequent due to associated renal (urolithiasis) or bone (osteitis fibrosa cystica) complications. A benign tumor of the parathyroid gland (single
\end{abstract}

Fecha de recibido: 07/06/2020 - Fecha de aceptación: 14/08/2020

Correspondencia: Aníbal Ariza Gutiérrez, Carrera 7 \# 40-62, Departamento de cirugía, Hospital Universitario San Ignacio, Bogotá, D.C., Colombia. Teléfono: +57 3176471466. Correo electrónico: anibal_ariza@hotmail.com

Citar como: Sánchez-De Guzmán G, Ariza A. Hiperparatiroidismo primario: conceptos para el cirujano general. Rev Colomb Cir. 2021;36:109-18. https://doi.org/10.30944/20117582.688

Este es un artículo de acceso abierto bajo una Licencia Creative Commons - BY-NC-ND https://creativecommons.org/licenses/by-ncnd/4.0/deed.es 
adenoma) is the main cause of this disease. Therefore, its treatment is usually surgical. Despite this, the management of this pathology by the general surgeon is not frequent. This article reviews key concepts for the diagnosis and management of this disease for the resident physician and specialist in General Surgery.

Keywords: parathyroid glands; hyperparathyroidism, primary; parathyroid hormone; parathyroid neoplasms; adenoma; hypercalcemia; parathyroidectomy.

\section{Introducción}

La primera descripción de las glándulas paratiroideas como órgano fue hecha en 1850 en el zoológico de Londres durante una necropsia a un rinoceronte. La primera cirugía de paratiroides fue realizada en 1925 en Viena por Félix Mandl en un paciente con una osteítis fibrosa quística. En ese mismo año, el bioquímico James B Collip, en Canadá, descubrió la hormona paratiroidea, (PTH, por sus siglas en inglés) después de participar en el grupo de investigadores que aislaron la insulina y la hormona adrenocorticotrópica (ACTH).

El hiperparatiroidismo primario (HPTP), es el tercer trastorno endocrino en frecuencia ${ }^{1}$, que se caracteriza porque, en ausencia de un estímulo reconocido, una o varias glándulas paratiroideas secretan en exceso PTH, resultando en hipercalcemia. Se estima que su prevalencia es de $0,85 \%$ y su incidencia actual es de aproximadamente 50 casos por cada 100.000 personas-año en Estados Unidos, siendo más frecuente en mujeres (2 a 3:1) entre 50-65 años de edad ${ }^{2}$.

La presentación clínica ha variado en los últimos 40 años, desde cuadros muy sintomáticos asociados a hipercalcemia severa, litiasis renal y enfermedad ósea (osteítis fibrosa quística), hasta una condición asintomática, más frecuente en la actualidad, diagnosticada de forma incidental con exámenes rutinarios (calcio sérico, pruebas bioquímicas, densitometría ósea, etc.)

\section{Embriología y anatomía}

Las glándulas paratiroideas se desarrollan entre la quinta y séptima semana de gestación. Las glándulas superiores se originan de la cuarta bolsa branquial y generalmente se localizan hacia la unión cricotraqueal, entre el tejido tiroideo y la rodilla del nervio laríngeo recurrente, mientras las glándulas inferiores se originan de la tercera bolsa branquial. Aproximadamente el $44 \%$ se encuentran en el ligamento tirotímico, el $26 \%$ en el cuerno tímico, el $17 \%$ en el polo inferior de la glándula tiroidea, $2 \%$ en el mediastino y el $11 \%$ están distribuidas en el lecho carotídeo superior o dentro del tiroides ${ }^{3}$.

En el $91 \%$ de las personas son 4 glándulas, en el $5 \%$ son 3 y en el $4 \%$ son 5 o más. Habitualmente cada una mide entre 5 y $7 \mathrm{~mm}$. Todo el tejido paratiroideo puede pesar entre 90 y 130 mg. En el $83 \%$ de los casos tienen forma ovalada, en $11 \%$ elongada, $5 \%$ arriñonada y $1 \%$ multilobulada. Generalmente se acompañan de tejido graso periglandular y en el $80 \%$ de los casos su ubicación contralateral es en espejo ${ }^{3}$. Usualmente las paratiroides reciben su irrigación de la arteria tiroidea inferior, sin embargo, hasta en un $45 \%$ de los pacientes, las glándulas superiores pueden recibir ramas de la arteria tiroidea superior ${ }^{4}$.

\section{Homeostasis del calcio}

La ingesta diaria de calcio en promedio es de 500 a $1000 \mathrm{mg}$. Su absorción ocurre principalmente en el duodeno y yeyuno proximal. En el riñón, casi todo el calcio filtrado se reabsorbe a nivel del túbulo proximal y el asa de Henle, a través de la bomba de sodio. En el túbulo distal, la absorción del calcio está mediada por la PTH. Las pérdidas diarias de calcio son aproximadamente de $100 \mathrm{mg}$ por transpiración y $800 \mathrm{mg}$ en las heces. El mayor depósito corporal de calcio está en los huesos, que contiene aproximadamente $1000 \mathrm{gr}^{5}$.

El control de los niveles séricos de calcio y fósforo es complejo, porque no sólo depende de la ingesta, sino de la participación de órganos como intestino (donde ocurre la absorción mediada por metabolitos de la vitamina D), riñón (excreción), 
hígado (fragmentación de la PTH), hueso (depósito y liberación), piel (activación de la vitamina $\mathrm{D}_{3}$ por irradiación ultravioleta del 7-dihidroxicolesterol), glándulas paratiroideas (hormona paratiroidea) y glándula tiroides (donde las células C o parafoliculares producen Calcitonina, la cual disminuye la reabsorción ósea por su efecto antagónico a la PTH).

Las concentraciones de calcio sérico normalmente deben mantenerse en un rango muy estrecho y preciso, entre 8,5-10,5 mg/dl o 2,1-2,6 $\mathrm{mmol} / \mathrm{l}$, que es el valor que se requiere para la realización óptima de múltiples procesos fisiológicos, intra y extracelulares en el organismo. Lograr la regulación de su concentración con exactitud, depende de la relación entre la absorción intestinal, la excreción renal y la captación y liberación ósea, procesos que a su vez están regulados por la acción de la hormona paratiroidea, la vitamina D3 y, en menor participación, de la calcitonina.

La PTH es la principal hormona reguladora de la homeostasis del calcio. Es producida por las células principales de la glándula paratiroides, en forma de una prohormona de 110 aminoácidos, que se divide y se convierte finalmente en la hormona propiamente dicha, un polipéptido de 84 aminoácidos. Tiene una porción biológicamente activa que corresponde al grupo amino $\mathrm{N}$-terminal (residuos 1-34) y una inactiva que corresponde al grupo carboxilo C-terminal (residuos 35 a 84). La vida media de la PTH intacta es de aproximadamente 6 minutos en el fragmento $\mathrm{N}$-terminal y casi 1 hora en el fragmento C-terminal. Su degradación ocurre principalmente en el hígado.

El calcio constituye el principal mineral corporal, pero solamente su forma ionizada es fisiológicamente activa. En suero se presenta $47 \%$ en forma ionizada, $45 \%$ ligado a proteínas y $8 \%$ en forma de aniones orgánicos. Su función es estimular la contracción muscular, la excitabilidad nerviosa, la formación de matriz ósea y facilitar la transducción de señales ${ }^{5}$.

La hipocalcemia es el principal estímulo fisiológico de las células principales en las glándulas paratiroideas, donde mediante tres receptores se regula la síntesis y secreción de la hormona paratiroidea:
1. El receptor de la vitamina D (RVD) que, al unirse a ésta, disminuye la síntesis de PTH

2. El receptor específico del calcio (RECa) que, al ser ocupado por este elemento, inhibe la síntesis y secreción de la PTH

3. El receptor del Factor de Crecimiento Fibroblástico (R-FCF) y la proteína transmembrana "Klotho" forman un complejo que es estimulado por el Factor de Crecimiento Fibroblástico 23 (FCF-23). El FCF-23 es una hormona que se produce en los osteocitos y osteoblastos cuando aumentan los niveles de fosfato y de calcitriol. El FCF-23 inhibe la secreción de PTH en las glándulas paratiroideas y disminuye la reabsorción de fosfato en el túbulo proximal del riñón.

La PTH actúa en el hueso sobre los osteocitos, osteoclastos y osteoblastos, incrementando los niveles de calcio. En el riñón promueve la retención de calcio y excreción de fosfato, y en el intestino, estimula la absorción del calcio.

A nivel del túbulo proximal renal, normalmente se realiza un intercambio recíproco entre el calcio y el fósforo para mantener sus niveles estables. Así mismo la $1 \alpha$-hidroxilasa permite la activación de la vitamina $\mathrm{D}$ (calcitriol). La acción de la $1 \alpha$-hidroxilasa es suprimida por el FCF-23. La hipocalcemia e hiperfosfatemia estimulan la producción de $\mathrm{PTH}$, mientras la vitamina D la inhibe ${ }^{6-8}$ (tabla 1 ).

\section{Fisiopatología y causas del hiperparatiroidismo primario}

En el HPTP el trastorno metabólico se localiza en las glándulas paratiroideas por pérdida en el control de la síntesis y secreción de la PTH. En más del $80 \%$ de los casos se detecta hipercalcemia asintomática, asociada a elevación de la PTH. Sin embargo, la hipercalcemia también puede presentarse con valores normales o mínimamente elevados de PTH. El HPTP normocalcémico ${ }^{8}$ es una de las formas de presentación del HPTP, en el que existen, en pruebas repetidas, niveles elevados de PTH con calcio sérico normal. Para su 
Tabla 1. Mecanismos principales de control del calcio, fósforo y vitamina D

\begin{tabular}{|c|c|c|c|c|}
\hline & Glandula paratiroidea & Riñón & Hueso & Intestino \\
\hline Estimulo & $\begin{array}{l}\text { Hipocalcemia e } \\
\text { hiperfosfatemia }\end{array}$ & $\begin{array}{l}\text { Hipocalcemia, hiperfosfatemia } \\
\text { y elevación de PTH }\end{array}$ & $\begin{array}{l}\text { Hiperfosfatemia y eleva- } \\
\text { ción de } 1,25 \text { Vit D3 }\end{array}$ & Hipocalcemia \\
\hline Respuesta & Elevación PTH & $\begin{array}{l}\text { Aumenta síntesis de } 1,25 \text { Vit } \\
\text { D3 }\end{array}$ & Liberación FCF-23 & \\
\hline \multirow{2}{*}{ Acción } & FCF-23: disminuye PTH & $\begin{array}{l}\text { FCF-23: Disminuye } 1,25 \text { Vit } \\
\text { D3, aumenta reabsorción de } \\
\text { calcio y excreción de fósforo }\end{array}$ & \multirow[t]{2}{*}{$\begin{array}{l}\text { PTH: Libera calcio y fós- } \\
\text { foro y aumenta síntesis } \\
\text { de FCF-23 }\end{array}$} & \multirow[t]{2}{*}{$\begin{array}{l}\text { 1,25Vit D3: Aumenta } \\
\text { absorción de calcio y } \\
\text { fósforo }\end{array}$} \\
\hline & $\begin{array}{l}\text { 1,25Vit D3: disminuye } \\
\text { PTH }\end{array}$ & $\begin{array}{l}\text { PTH: Aumenta } 1,25 \mathrm{Vit} \mathrm{D} 3 \text {, au- } \\
\text { menta reabsorción de calcio y } \\
\text { excreción }\end{array}$ & & \\
\hline
\end{tabular}

* PTH: hormona paratiroidea, 1,25Vit $\mathrm{D}_{3}$ : calcitriol, FCF-23: factor de crecimiento fibroblástico-23.

diagnóstico, se deben excluir otras causas de hiperparatiroidismo secundario, como enfermedad renal, deficiencia de vitamina $\mathrm{D}$, medicamentos o síndromes de malabsorción, entre otros.

La hipercalcemia hipocalciúrica familiar (HHF), una condición benigna hereditaria, es uno de los diagnósticos diferenciales principales del HPTP. Se presenta en individuos jóvenes, con hipercalcemia crónica y niveles de PTH normales o levemente elevados ${ }^{9}$. Según las guías clínicas, el cociente de aclaramiento calcio/creatinina (CCCR) es el índice bioquímico de elección para diferenciar entre HPTP y HHF. Un cociente menor de 0,01 es sugestivo de HHF y uno mayor de 0,02 , de HPTP. Los pacientes con HHF no requieren tratamiento quirúrgico ${ }^{10}$.

En aproximadamente el $86 \%$ de los casos de HPTP, la causa es un adenoma único de paratiroides, en el $9 \%$ hiperplasia difusa o nodular de una o varias glándulas, en el $3 \%$ adenoma doble y en el 1-2 \% carcinoma.

Cuando se trata de un carcinoma, generalmente hay órganos blanco comprometidos, por lo que se presenta osteoporosis, fractura patológica, urolitiasis, pancreatitis, o trastorno de la conciencia. En el $75 \%$ de los casos el nivel de calcio sérico está por encima de $14 \mathrm{mg} / \mathrm{dl}$ y la PTH intacta elevada más de 4 veces su valor normal. Puede cursar con parálisis del pliegue vocal, en el $50 \%$ de los casos se palpa nódulo en el cuello y en el $75 \%$ su tamaño es mayor de $2 \mathrm{~cm}^{11,12}$.

\section{Otras causas de Hiperparatiroidismo primario}

\section{Síndromes hereditarios}

En un $95 \%$ de los casos, el HPTP es de presentación esporádica ${ }^{13}$; sin embargo, en muy raras ocasiones se presenta como parte de síndromes familiares, en edades tempanas. El hiperparatiroidismo familiar incluye un grupo de desórdenes en donde el HPTP es heredado, usualmente, como una característica autosómica dominante.

El síndrome de neoplasia endocrina múltiple (NEM) tipo 1, es causado por mutaciones inactivadoras en el gen NEM1 (11q13) que codifica la proteína menina, un supresor tumoral. Inicialmente descrito en 1954, es la causa más común de HPTP familiar, representando aproximadamente $2-4 \%$ de todos los $\operatorname{casos}^{13}$. Se caracteriza por una predisposición a desarrollar tumores endocrinos a nivel de la hipófisis, paratiroides y tumores neuroendocrinos pancreáticos y del tracto gastrointestinal. La asociación de la mutación del gen NEM1 con la presentación esporádica o familiar de adenomas paratiroideos ha sido bien documentada, mientras que su asociación con el carcinoma paratiroideo es rara. El HPTP es el trastorno endocrino más frecuente, presente en alrededor del $90 \%$ de los pacientes entre 20 y 25 años y generalmente hay compromiso multiglandular con crecimiento de todas las glándulas. 
La NEM tipo 4, más recientemente descrita, es similar al NEM1. Los afectados desarrollan tumores paratiroideos, hipofisiarios, pancreáticos, adrenales y, en raras ocasiones, de cérvix y testículos. Es causado por mutaciones inactivadoras en el gen $C D K N 1 B$, que codifica la proteína p27, la cual actúa como un regulador de la progresión del ciclo celular. Más de un $80 \%$ de los afectados por este síndrome, presentan hiperparatiroidismo primario ${ }^{14}$.

El síndrome NEM tipo 2A, causado por mutaciones en el protooncogen RET en el cromosoma 10 , se caracteriza por el hallazgo de carcinoma medular de tiroides, feocromocitoma e hiperplasia o adenoma de la paratiroides. El HPTP ocurre en un 20-30\% de los casos, usualmente con presentación clínica leve o asintomática. La progresión maligna de los adenomas paratiroideos es rara ${ }^{13}$.

El síndrome de hiperparatiroidismo por tumor mandibular (HPT-TM) es causado por mutaciones en el gen HPRT2 en el cromosoma 1, que originan tumores paratiroideos, fibromas osificantes de la mandíbula, lesiones renales (tumor de Wilms, carcinoma papilar renal, enfermedad quística) e HPTP ${ }^{14}$. En más del $95 \%$ de los pacientes, la primera manifestación es el hiperparatiroidismo primario, con un comportamiento más agresivo por hipercalcemia severa y con frecuencia inusualmente alta de carcinoma paratiroideo $(10-15 \%)^{13}$.

Otro de los síndromes hereditarios, es el Hiperparatiroidismo Familiar Aislado, muy poco frecuente, que se caracteriza por la presencia de hiperparatiroidismo primario causado por tumores únicos o múltiples paratiroideos, en al menos 2 familiares de primer grado y en ausencia de otros tumores o trastornos endocrinos. No se conoce el mecanismo especifico, pero se han detectado mutaciones en el gen NEM1 (20-23\%), en el gen RECa (receptor especifico de calcio, en 14-18\%) y en el gen HRTPT2, en menor porcentaje (tabla 2).

Tabla 2. Características de síndromes hereditarios en hiperparatiroidismo primario

\begin{tabular}{|c|c|c|c|c|c|}
\hline Caracteristicas & NEM 1 & NEM 2A & NEM 4 & HPT-TM & HPT familiar \\
\hline \multicolumn{6}{|l|}{ Hiperparatiroidismo } \\
\hline \multicolumn{6}{|c|}{ Tumores pancreáticos } \\
\hline \multicolumn{6}{|c|}{ Adenomas hipofisiarios } \\
\hline \multicolumn{6}{|c|}{ Cáncer medular de tiroides } \\
\hline \multicolumn{6}{|l|}{ Feocromocitoma } \\
\hline \multicolumn{6}{|c|}{ Tumores de mandíbula y renales } \\
\hline Herencia & $A D$ & $A D$ & $A D$ & $A D$ & $A D$ \\
\hline Mutación de genes & NEM 1 & $\begin{array}{l}\text { RET proto } \\
\text { oncogene }\end{array}$ & CDKN1B & HRPT2 & $\begin{array}{c}\text { NEM1-RECa- } \\
\text { HRPT2 }\end{array}$ \\
\hline
\end{tabular}

* NEM: neoplasia endocrina múltiple, HPT: hiperparatiroidismo, HPT-TM: hiperparatiroidismo - tumor mandibular, AD: autosómico dominante, CDKN1B: inhibidor 1B de quinasa dependiente de ciclina, RECa: receptor específico del calcio. 


\section{Exposición a radiación}

Pacientes con HPTP pueden tener historia de irradiación a cabeza y cuello, en un rango de 20 a 40 años previos al desarrollo de la condición. El ejemplo más representativo, es una cohorte de 61 trabajadores de la planta nuclear en Chernóbil en 1986 donde 15 de ellos desarrollaron HPTP. La exposición media fue entre 0,3 a 8,7 Gy. No se ha demostrado diferencias en el curso clínico o recurrencia entre pacientes expuestos y no expuestos. Sin embargo, en pacientes expuestos es más frecuente la asociación con tumores tiroideos ${ }^{15}$.

\section{Medicamentos}

La terapia con litio y diuréticos tiazídicos se han asociado como factores de riesgo para el HPTP ${ }^{16}$. La experiencia más reciente con las tiazidas ha sugerido que la hipercalcemia, en este contexto, posiblemente enmascara el estado subyacente del HPTP, y no es probable que se revierta cuando se suspende el diurético ${ }^{17}$.

\section{Diagnóstico}

La mayoría de los casos de HPTP son diagnosticados incidentalmente por laboratorios de rutina o dentro del seguimiento de osteopenia o urolitiasis. Clínicamente, se presenta como hipercalcemia asintomática en alrededor de $80 \%$ de los casos.

El HPTP sintomático puede manifestarse con dolores óseos, fatiga, pérdida de peso, úlcera pép- tica, pancreatitis, nefrolitiasis, arterioesclerosis acelerada, déficit cognitivo y ansiedad.

Los laboratorios frecuentemente muestran elevación del calcio y PTH séricos, y aumento en la excreción urinaria de calcio, y en las radiografías óseas se observa el patrón típico de "gránulos de sal y pimienta", compatible con osteítis fibrosa quística, osteopenia del tercio distal de la clavícula, reabsorción subperióstica de las falanges distales, quistes óseos o tumores pardos.

La gammagrafía realizada con Tc99m(tecnecio)-Sestamibi, tanto en imágenes planares como de tomografía computarizada por emisión de fotón único (SPECT, por sus siglas en ingles), tiene utilidad en la localización de adenomas paratiroideos, especialmente en aquellos de tamaño superior a $500 \mathrm{mg}^{18,19}$. La sensibilidad y la especificidad mejora cuando las imágenes de SPECT son fusionadas con imágenes de la tomografía computarizada (figura 1), preferiblemente adquiridas en equipos híbridos, pasando la sensibilidad de $83 \%$ a $96 \%$ y la especificidad de 80 a $93 \%$, ventajas más evidentes en adenomas de menor tamaño, de alrededor de $210 \mathrm{mg}^{20}$. La sensibilidad es menor (58\%) en el caso de glándulas hiperplásicas ${ }^{21}$. Cuando las imágenes de medicina nuclear convencional (SPECT) son negativas, una alternativa es realizar una tomografía por emisión de positrones (en inglés, PET-CT) con C11-Metionina, con F18-Colina o C11-Colina ${ }^{22,23}$.
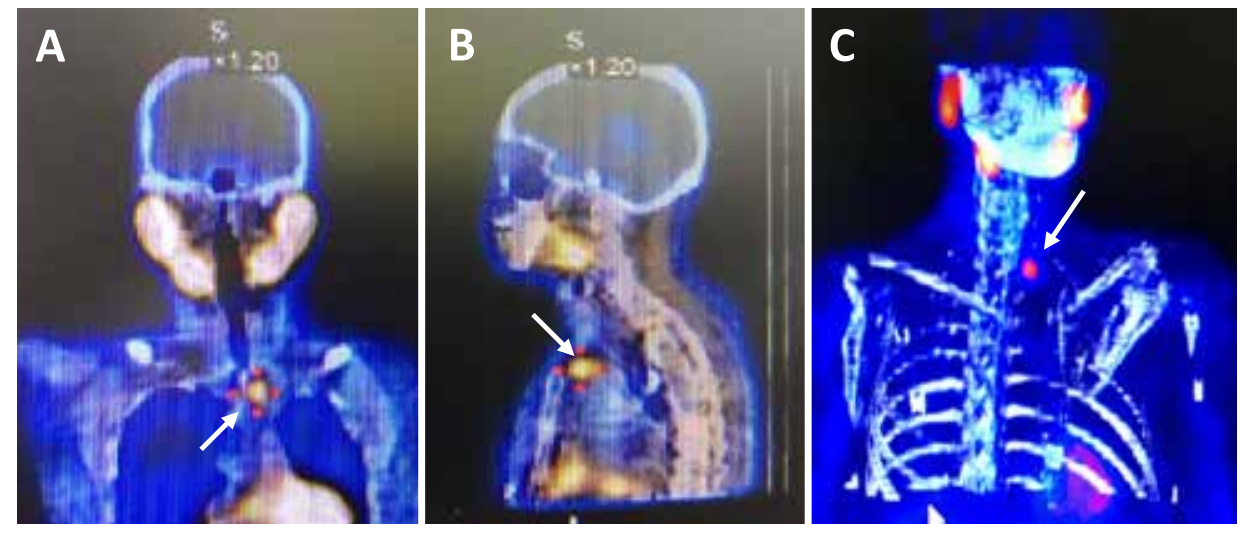

Figura 1. Gammagrafía de paratiroides con SPECT-CT que muestra hipercaptación de la glándula paratiroidea inferior izquierda (flechas blancas). 
Es recomendable tener una ecografía de tiroides para descartar patología tiroidea concomitante. Otras imágenes como la tomografía contrastada, resonancia magnética, angiografía con muestreo selectivo de PTH o PETscan, sólo se utilizan en caso de gammagrafía negativa, recidiva o difícil localización de la glándula o las glándulas enfermas y de tejido paratiroideo ectópico ${ }^{24}$.

Como se explicó previamente, un diagnóstico diferencial es la hipercalcemia hipocalciúrica familiar benigna, que cursa con niveles normales o ligeramente elevados de PTH. Generalmente se diagnostica por persistencia de hipercalcemia posterior a paratiroidectomía parcial ${ }^{25}$.

Aunque se sospeche carcinoma primario de paratiroides, no es recomendable realizar biopsia con aguja fina, por su baja especificidad y por la posibilidad de desarrollar paratiromatosis.

\section{Tratamiento}

La cirugía es el tratamiento recomendado en el HPTP (tabla 3), para el paciente, sintomático o asintomático, que tenga compromiso de órgano blanco (osteopenia, urolitiasis, disminución de la tasa de filtración glomerular, úlcera péptica, pancreatitis o trastorno neuromuscular).

Un cirujano experimentado está en capacidad de identificar la o las glándulas afectadas en el $95 \%$ de los casos. La fusión de imágenes (SPECT-CT) y la medición de la PTHi (molécula intacta) en el transcurso de la cirugía, han permitido tomar decisiones terapéuticas más acertadas, disminuyendo la tasa de recidiva del

Tabla 3. Indicaciones de manejo quirúrgico del hiperparatiroidismo primario.

\begin{tabular}{l}
\hline - Paciente menor de 50 años \\
\hline - Calcio sérico mayor de $12 \mathrm{mg} / 24$ horas \\
\hline - Calciuria mayor de $400 \mathrm{mg} / 24$ horas \\
\hline - Disminución de la depuración de creatinina mayor del \\
$30 \%$ para la edad \\
\hline - Densitometría lumbar, de cadera o radio distal con \\
T-score menor de -2.5 \\
\hline - Pacientes con imposibilidad de seguimiento medico
\end{tabular}

HPTP a menos del $5 \%{ }^{26}$. La cirugía radioguiada y la decisión de realizar abordaje invasivo mínimo dependerá de la tecnología disponible en cada centro médico y de la experiencia del cirujano (figuras 2 y 3 ).

Habitualmente es un procedimiento ambulatorio, el paciente egresa con suplementos de calcio (3 gr/día) y calcitriol $(0.25 \mathrm{mcg} /$ día). Se realiza control en 3 semanas con nuevos valores de PTHi, fosfatasa alcalina, calcio y fósforo séricos.

En pacientes de alto riesgo quirúrgico, o que rechazan el procedimiento, se debe recomendar un seguimiento anual estricto con niveles de cal-

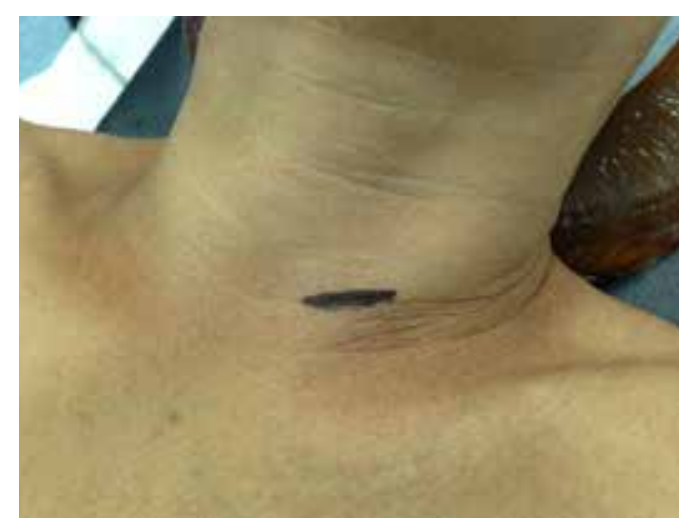

Figura 2. Abordaje quirúrgico por vía retro-tiroidea izquierda

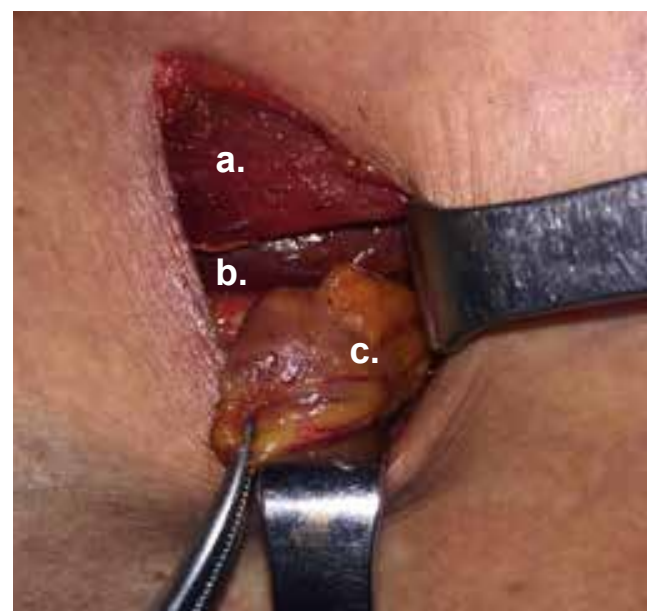

Figura 3. Adenoma de paratiroides inferior izquierda. a. Músculo esternotiroideo; b. lóbulo tiroideo izquierdo; c. adenoma de paratiroides. 
cio, creatinina y densitometría ósea. En algunos casos con hipercalcemia sintomática se recomienda mantener buena hidratación, restricción de la ingesta de calcio, asegurar niveles normales de vitamina $\mathrm{D}$, e incluso, el uso de calciomiméticos. Es recomendable que la decisión terapéutica sea tomada entre el cirujano, el endocrinólogo y el paciente ${ }^{27-29}$.

En el carcinoma primario se recomienda cirugía radical (previa corrección de la hipercalcemia severa), con hemitiroidectomía ipsilateral, exploración contralateral y hasta el timectomía, con linfadenectomía en caso de compromiso histológico documentado.

\section{Complicaciones del manejo quirúrgico}

La complicación más frecuente es la hipocalcemia transitoria, que se presenta entre el $15 \%$ y el $30 \%$ de los pacientes, y representa el $7 \%$ de reingresos en postoperatorio para administración endovenosa de calcio. Cuando el paciente ingresa por urgencias y se documenta hipocalcemia sintomática, la administración de calcio debe ser simultáneamente oral y endovenosa. Se recomienda infusión de gluconato de calcio 2 ampollas en 100 cc de solución salina normal o en dextrosa en agua, para pasar en 15 minutos (no se debe usar lactato de ringer por la precipitación del gluconato), y continuar con 1 ampolla cada 6 horas hasta controlar los síntomas. El calcio oral se administra 1.5-3 gr cada 6 horas, en presentación de carbonato o citrato, y el calcitriol $0.25 \mathrm{mcg}$ cada 8 horas. Con este manejo se logra el egreso del paciente en 12 a 24 horas en la gran mayoría de casos.

En algunos casos, la hipocalcemia postoperatoria es severa y prolongada, a pesar de tener una PTH normal o ligeramente elevada, y se puede asociar a hipofosfatemia, hipomagnesemia e hipercalemia, requiriendo manejo hospitalario con monitoreo cardiaco, suplemento oral y endovenoso de calcio, magnesio y calcitriol. Esta condición se conoce como "síndrome del hueso hambriento" y se presenta en aproximadamente un $13 \%$ de los pacientes llevados a paratiroidectomia por HPTP. Generalmente afecta a pacientes con osteítis fibrosa y a pacientes que han recibido terapia con calciomiméticos por un tiempo prolongado. También se consideran factores de riesgo para desarrollarlo, la edad menor de 45 años, obesidad, niveles altos de fosfatasa alcalina y niveles normales o bajos de calcio sérico preoperatorios 8,30,31. En instituciones con alto volumen de cirugía de paratiroides y con recursos, han utilizado la criopreservación de tejido paratiroideo con el fin de reimplantarlo en caso de hipoparatiroidismo severo de difícil manejo ${ }^{32}$.

El sangrado, la infección o la lesión del nervio laríngeo recurrente se presentan aproximadamente en el $1 \%$ de los casos. La mortalidad perioperatoria es del $0,3 \%{ }^{33-35}$.

La complicación menos deseada por el cirujano es la imposibilidad de encontrar la o las glándulas enfermas y la consecuente persistencia del hiperparatiroidismo. Generalmente las fallas se presentan en la localización preoperatoria, en la exploración cervical o por subestimar el número de glándulas paratiroideas con hiperplasia versus adenomas. En la serie reportada por Tominaga Y., informó una incidencia de HPT persistente del $4 \%$ y una tasa de reintervención por esta causa, del $1,6 \%{ }^{35}$.

La paratiromatosis es la siembra de múltiples focos de tejido paratiroideo benigno e hiperfuncionante en los tejidos blandos del cuello o mediastino, producto de la ruptura de la cápsula de la glándula paratiroidea durante la exploración quirúrgica, de la realización de biopsia con aguja fina, o del uso percutáneo de la escleroterapia con alcohol. Estos dos últimos procedimientos no son recomendados de rutina ${ }^{35}$.

\section{Conclusiones}

El hiperparatiroidismo primario, es una causa común de hipercalcemia. Su presentación clínica ha evolucionado hasta una condición mayormente asintomática en la población, cuyo diagnóstico se hace a partir de exámenes bioquímicos rutinarios. En pacientes jóvenes, su abordaje diagnóstico debe ser más cuidadoso; en estos casos puede ser un componente de múltiples anormalidades endocrinológicas hereditarias que, si bien representan menos del $5 \%$ del HPTP, afectan con mayor 
frecuencia a este grupo etáreo. La cirugía es el único tratamiento curativo de esta enfermedad; se recomienda en pacientes jóvenes sintomáticos, o en aquellos asintomáticos que están en riesgo de presentar compromiso de órgano blanco. En la actualidad, el estudio prequirúrgico con imágenes diagnósticas (gammagrafía o SPECT) permite localizar el adenoma con mayor precisión, evitando recidivas y permitiendo abordajes de invasión mínima.

\section{Consideraciones éticas}

Consentimiento informado: Este estudio es una revisión de la literatura, y como tal no hay necesidad de un consentimiento informado ni de aprobación del Comité de Ética Institucional.

Conflictos de interés: Los autores declaramos no tener conflictos de interés.

Fuente de financiación: El presente trabajo fue autofinanciado.

Contribución de los autores: Concepción y diseño del estudio, adquisición, análisis e interpretación de datos, redacción, revisión crítica y aprobación final del manuscrito: Gabriel Sánchez-De Guzmán y Aníbal Ariza.

\section{Referencias}

1. Madkhali T, Alhefdhi A, Chen H, Elfenbein D. Primary hyperparathyroidism. Turkish J Surg. 2016;32:58-66. https://doi.org/10.5152/UCD.2015.3032

2. Griebeler ML, Kearns AE, Ryu E, Hathcock MA, Melton LJ, Wermers RA. Secular trends in the incidence of primary hyperparathyroidism over five decades (19652010). Bone. 2015;73:1-7. https://doi.org/10.1016/j.bone.2014.12.003

3. Akerstrom G, Malmaeus J, Bergstrom R. Surgical anatomy of human parathyroid glands. Surgery. 1984;95:14-21.

4. Arrangoiz R, Cordera F, Caba D, Muñoz-Juárez M, Moreno E, Luque E. Parathyroid embryology, anatomy, and pathophysiology of primary hyperparathyroidism. Int J Otolaryngol Head \& Neck Surg. 2017;06:39-58. https://doi.org/10.4236/ijohns.2017.64007

5. Bloom MB, Norton JA. Parathyroid. In: Norton J, Barie PS, Bollinger RR, Chang AE, Lowry S, Mulvihill SJ, et al, editors. Surgery Basic Science an Clinical Evidence. 2nd ed. Nueva York: Springer; 2008. p. 857-77.

6. Bellorin-Font E, Ambrosoni P, Carlini RG, Carvalho AB, Correa-Rotter R, Cueto-Manzano A, et al. Guías de prác- tica clínica para la prevención, diagnóstico, evaluación y tratamiento de los trastornos minerales y óseos en la enfermedad renal crónica (TMO-ERC) en adultos. Nefrologia. 2013;33(SUPPL1):1-28.

https://doi.org/10.3265/Nefrologia.pre2013.Feb. 11945

7. Cunningham J, Locatelli F, Rodriguez M. Secondary hyperparathyroidism: Pathogenesis, disease progression, and therapeutic options. Clin J Am Soc Nephrol. 2011;6:913-21.

https://doi.org/10.2215/CJN.06040710

8. Lau WL, Obi Y, Kalantar-Zadeh K. Parathyroidectomy in the management of secondary hyperparathyroidism. Clin J Am Soc Nephrol. 2018;13: 952-61. https://doi.org/10.2215/CJN.10390917

9. Blackburn M, Diamond T. Primary hyperparathyroidism and familial hyperparathyroid syndromes. Aust Fam Physician. 2007;36:1029-33.

10. Lee JY, Shoback DM. Familial hypocalciuric hypercalcemia and related disorders. Best Pract Res Clin Endocrinol Metab. 2018;32:609-19.

https://doi.org/10.1016/j.beem.2018.05.004

11. Myers E, Suen Y, Watson C. Cancer of the parathyroid. In: Myers E, Suen Y, eds. Cancer of the Head and Neck. 3th ed. Philadelphia: W.B Saunders; 1996. p. 516-24.

12. Rodríguez-Portillo M, Rodríguez-Ortiz ME. Secondary hyperparthyroidism: Pathogenesis, diagnosis, preventive and therapeutic strategies. Rev Endocr Metab Dis. 2017;18:79-95. https://doi.org/10.1007/s11154-017-9421-4

13. Giusti F, Cavalli L, Cavalli T, Brandi ML. Hereditary hyperparathyroidism syndromes. J Clin Densitom. 2013;16:69-74.

http://doi.org/10.1016/j.jocd.2012.11.003

14. Falchetti A. Genetics of parathyroids disorders: Overview. Best Pract Res Clin Endocrinol Metab. 2018;32:781-90. https://doi.org/10.1016/j.beem.2018.09.011

15. Boehm BO, Rosinger S, Belyi D, Dietrich JW. The Parathyroid as a target for radiation damage. N Engl J Med. 2011;365:676-8. https://doi.org/10.1056/NEJMc1104982

16. Szalat A, Mazeh H, Freund HR. Lithium-associated hyperparathyroidism: Report of four cases and review of the literature. Eur J Endocrinol. 2009;160:317-23. https://doi.org/10.1530/EJE-08-0620

17. Griebeler ML, Kearns AE, Ryu E, Thapa P, Hathcock MA, Melton LJ, Wermers RA. Thiazide-associated hypercalcemia: Incidence and association with primary hyperparathyroidism over two decades. J Clin Endocrinol Metab. 2016;101:1166-73. https://doi.org/10.1210/jc.2015-3964

18. Greenspan BS, Dillehay G, Intenzo C, Lavely WC, O’Doherty $\mathrm{M}$, Palestro CJ, et al. SNM practice guideline for 
parathyroid scintigraphy 4.0. J Nucl Med Technol. 2012;40:111-8.

https://doi.org/10.2967/jnmt.112.105122

19. Hindié E, Ugur Ö, Fuster D, O’Doherty M, Grassetto G, Ureña P, et al. 2009 EANM parathyroid guidelines. Eur J Nucl Med Mol Imaging. 2009;36:1201-16. https://doi.org/10.1007/s00259-009-1131-z

20. Sandqvist P, Nilsson IL, Grybäck P, Sanchez-Crespo A, Sundin A. SPECT/CT's advantage for preoperative localization of small parathyroid adenomas in primary hyperparathyroidism. Clin Nucl Med. 2017;42:e109-14. https://doi.org/10.1097/RLU.0000000000001447

21. Caldarella C, Treglia G, Pontecorvi A, Giordano A. Diagnostic performance of planar scintigraphy using 99mTc-MIBI in patients with secondary hyperparathyroidism: A meta-analysis. Ann Nucl Med. 2012;26:794-803.

https://doi.org/10.1007/s12149-012-0643-y

22. Yuan L, Liu J, Kan Y, Yang J, Wang X. The diagnostic value of 11C-methionine PET in hyperparathyroidism with negative 99mTc-MIBI SPECT: A meta-analysis. Acta Radiologica. 2017;58:558-64. https://doi.org/10.1177/0284185116661878

23. Orevi M, Freedman N, Mishani E, Bocher M, Jacobson 0 , Krausz Y. Localization of parathyroid adenoma by ${ }^{11} \mathrm{C}$-Choline PET/CT: preliminary results. Clin Nucl Med. 2014;39:1033-8. https://doi.org/10.1097/RLU.0000000000000607

24. Walker MD, Silverberg SJ. Primary hyperparathyroidism. Nat Rev Endocrinol. 2018;14:115-25. https://doi.org/10.1038/nrendo.2017.104

25. Fraser WD. Hyperparathyroidism. Lancet. 2009;374: 145-58. https://doi.org/10.1016/S0140-6736(09)60507-9

26. Khan AA, Hanley DA, Rizzoli R, Bollerslev J, Young JEM, Rejnmark L, et al. Primary hyperparathyroidism: review and recommendations on evaluation, diagnosis, and management. A Canadian and international consensus. Osteoporosis Int. 2017;28:1-19. https://doi.org/10.1007/s00198-016-3716-2
27. Bilezikian JP, Bandeira L, Khan A, Cusano NE. Hyperparathyroidism. Lancet. 2018;391:168-78. https://doi.org/10.1016/S0140-6736(17)31430-7

28. Bilezikian JP. Primary hyperparathyroidism. J Clin Endocrinol Metab. 2018;103:3993-4004. https://doi.org/10.1210/jc.2018-01225

29. Herrero JA, López-Gómez JM, Maduell F, Martín-De Francisco AL, Martín-Malo A, Martínez-Castelao A, et al. Activación de los receptores de vitamina D en la optimización del hiperparatiroidismo secundario en diálisis. Nefrologia. 2013;33:571-84.

https://doi.org/10.3265/Nefrologia.pre2013.May. 11901

30. Ho LY, Wong PN, Sin HK, Wong YY, Lo KC, Chan SF, et al. Risk factors and clinical course of hungry bone syndrome after total parathyroidectomy in dialysis patients with secondary hyperparathyroidism. BMC Nephrol. 2017;18:12. https://doi.org/10.1186/s12882-016-0421-5

31. Jakubauskas M, Beiša V, Strupas K. Risk factors of developing the hungry bone syndrome after parathyroidectomy for primary hyperparathyroidism. Acta Medica Litu. 2018;25:45-51. https://doi.org/10.6001/actamedica.v25i1.3703

32. Cohen MS, Dilley WG, Wells SA, Moley JF, Doherty GM, Sicard GA, et al. Long-term functionality of cryopreserved parathyroid autografts: A 13-year prospective analysis. Surgery. 2005;138:1033-41. https://doi.org/10.1016/j.surg.2005.09.029

33. Pitt SC, Sippel RS, Chen H. Secondary and tertiary hyperparathyroidism, state of the art surgical management. Surg Clin North Am. 2009;89:1227-39. https://doi.org/10.1016/j.suc.2009.06.011

34. Schlosser K, Zielke A, Rothmund M. Medical and surgical treatment for secondary and tertiary hyperparathyroidism. Scand J Surg. 2004;93:288-97. https://doi.org/10.1177/145749690409300407

35. Tominaga Y. Surgical treatment of secondary hyperparathyroidism due to chronic kidney disease. Ups J Med Sci. 2006;111:277-92.

https://doi.org/10.3109/2000-1967-047 